Aus der Chirurgischen Universitätsklinik zu Königsberg i. Pr. (Direktor: Prof. Dr. M. Kirschner.)

\title{
Verfahren zur Erzielung der Kontinenz bei Anus praeternaturalis.
}

Von Dr. Kurtzahn, Assistent der Klinik.

(Mit I I Abbildungen.)

Die Aufgabe der Wiederherstellung der Kontinenz bei Anus praeternaturalis wurde auf mannigfaltigen Wegen zu lösen versucht. S ch mied en unterscheidet 3 Grundprinzipien, welche zur Geltung gebracht oder auch miteinander kombiniert werden können :

I. Die Knickung des Darmes vor seiner Ausmündung,

2. die verschiedenen Methoden der mechanischen Verengerung und

3. Versuche einer muskulären Sphincterbildung.

Zunächst ist für die operative Behebung der Inkontinenz besonders die Stelle der Ausmündung des künstlichen Afters von Bedeutung. Sowohl für den Anus sacralis, wie für den Anus perinealis oder den gelähmten Sphincter wie für den Anus praeternaturalis iliacus sind Verfahren erdacht worden. Uns beschäftigt hier besonders die Frage der operativen Behebung der Inkontinenz des Anus iliacus.

Schon mit der Anlegung eines Anus iliacus ist nämlich ein gewisser Schritt zur Erzielung der Kontinenz geschehen; Pelotten halten den Stuhl, wenn auch nur unvollkommen, so doch wesentlich besser zurück, als dieses beim Anus sacralis oder perinealis geschieht; dazu gestattet der Anus iliacus dem Patienten, der seinen Kunstafter unter Augen hat, in viel größerem Maße die Säuberung und Hautpflege dieser Stelle. Diese Tatsachen haben uns bereits seit langer Zeit bestimmt, in jedem Falle von Amputatio recti, bei der der Sphincter geopfert werden mußte, einen Anus iliacus anzu- 
legen. Auf diesen beziehen sich demnach auch unsere Versuche zur Erzielung der Kontinenz.

Auch beim Anus iliacus treten die oben erwähnten drei Grundprinzipien für Kontinenzoperationen miteinander in Wettbewerb und auch hier ist die Zahl der Verfahren so groß, daß es zu weit führen würde, auf sie einzeln einzugehen. Es hieße das, die Schmiedensche Arbeit wiederholen. Bisher ist es keinem Operationsvorschlag gelungen, sich allgemeine Geltung zu verschaffen. Die ideale Lösung der Aufgabe wäre wohl die Herstellung des Sphincters durch eine Muskelplastik. Leider haben sich die vielfach angestellten Versuche in dieser Richtung (F ö de $\mathrm{r} l, \mathrm{~W}$ it z el, Rydygier, Schoemaker, Lennander) nicht als ermutigend erwiesen. Die besondere Funktion eines Sphincters und die Eigenart seines Tonus lassen sich nicht imitieren. „Ein willkürlicher Muskel kann sich nur einige Zeit tonisch kontrahieren, dann erlahmt er, und eine anerlernte isolierte Innervation eines neugebildeten Sphincters in dieser Art ist wohl nicht denkbar" (F ö d e r l).

Hier muß erwähnt werden, daß die Dinge beim Anus sacralis und perinealis etwas anders $z u$ liegen scheinen. Noch in letzter Zeit hat Jenkel über Erfolge der Schoemakerschen Operation berichtet. Auch Goebell hat diese Operation einmal ausgeführt, wobei er die Muskelbündel des Glutaeus maximus durch freie Fascienplastik verlängerte. Er erzielte ein gutes Resultat. K ü mmell weist darauf hin, daß es sich um einen sehr großen Eingriff handele, bei dem die Asepsis schwer zu wahren sei. Immerhin sind durch Muskelplastik beim künstlichen oder inkontinenten Dammafter mehr oder weniger große Erfolge erzielt worden. Beim Anus iliacus, der uns hier beschäftigt, liegen die Dinge jedoch anders; da gelten die vorher angeführten Bedenken in besonderem Maße, und Erfolg versprechen nur solche plastischen Operationsverfahren, welche die Kontinenz auf mechanischem Wege erstreben, entweder durch Knickung, Verengerung oder Torsion des ausmündenden Darmteiles (Gersuny) oder durch plastische Operationen, welche es gestatten, das Darmrohr mechanisch zusammenzudrücken. Aber auch hier hat keine der vorgeschlagenen Operationen völlig befriedigt. Im Gegenteil, das völlige Ausbleiben des Erfolges nach Ausführung solcher Operationen hat zu durchaus ablehnenden Urteilen über diese Operationen überhaupt ge- 
führt (Poppert, Lieblein, Hochenegg). Demgegenüber glaubt aber $\mathrm{Schmieden,} \mathrm{daß} \mathrm{man} \mathrm{auf} \mathrm{Grund} \mathrm{der} \mathrm{Literatur-}$ durchsicht doch unmöglich zu einem ganz allgemein absprechenden Urteil kommen könne und meint, daß es, wenn auch nicht regelmäßig, so doch häufig gelänge, Besserung oder Heilung herbeizuführen. Jedenfalls folgert daraus, daß die Frage der operativen Erzielung der Kontinenz noch nicht abgeschlossen ist. Jede neue Möglichkeit, die Inkontinenz des Anus iliacus zu beheben, oder doch wenigstens dem Patienten ohne Gefahr mehr zu nutzen als bisher, muß daher erprobt werden.

Ein neuer Weg wurde in der Heranziehung von Hautkanälen zu diesen plastischen Operationen beschritten. Theoretisch erschien es möglich, sowohl den Darm zwischen 2 Hautschläuchen zu komprimieren, als auch ihn zwischen der äußeren Haut und einem in die Tiefe verpflanzten Hautschlauch zusammenzudrücken (Kurtzahn). Die erstere Möglichkeit wurde der umständlicheren 'Technik wegen in praxi von uns nicht versucht. Wir beschränkten uns hier auf Leichenversuche. Kü mmell teilt aber kürzlich mit, $\mathrm{da} B$ er es unternommen habe, einen Abschluß des Anus mit mechanischen Klemmen zu erzielen. Das Ziel war, zu beiden Seiten des Anus epithelisierte Kanäle anzulegen, in welche auswechselbare Gummiringe eingelegt wurden. Sein Vorgehen dürfte unserem nur an der Leiche versuchten ersten Verfahren entsprechen. Wir haben uns nur der $z$ weiten Methode bedient, deren Prinzip aus Fig. I

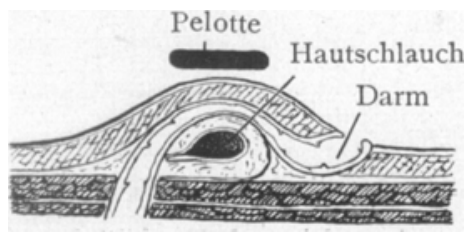

Fig. I.

deutlich hervorgeht. Der Darm wird hier zwischen einer Pelotte und einem im Hautschlauch befindlichen Gummistab zusammengedrückt.

Der Eingriff wurde durch Kirs ch ner erstmalig im Sommer I 919 vorgenommen. Es handelte sich um einen Patienten mit einem tiefsitzenden Rektumkarzinom. Die Amputatio recti wurde nach der kombinierten Methode ausgeführt, der Sphincter mußte 
entfernt werden. Die Operation verlief typisch. Durch eine besondere, lateral vom äußeren Rektusrande quer angelegte Öffnung wurde der Dickdarm etwa $10 \mathrm{~cm}$ heraushängen gelassen; unterhalb dieser Stelle wurde ein quadratisches Hautstück von etwa $7 \mathrm{~cm}$ Seitenlänge umschnitten und die untere Hälfte von der Unterlage abgelöst (Fig. 2). Wurde nun dieser untere Teil nach oben geklappt und die Schnittränder vernäht, so ergab sich ein Haut-

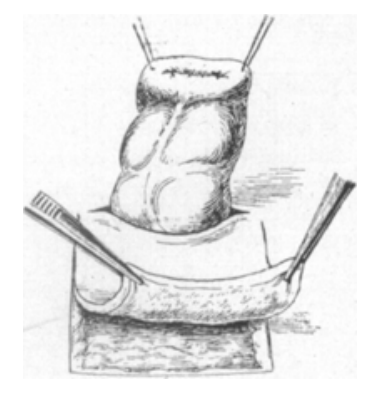

Fig. 2.

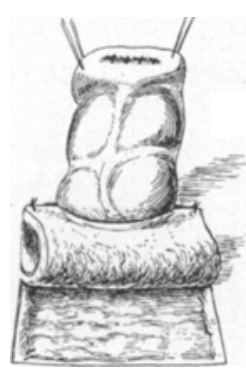

Fig. 3.

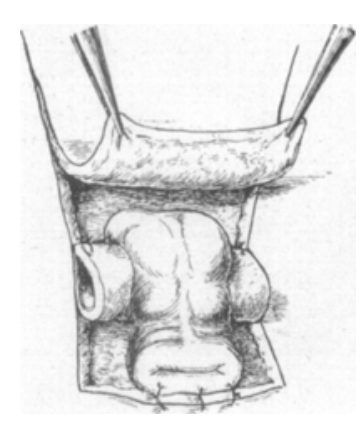

Fig. 4.

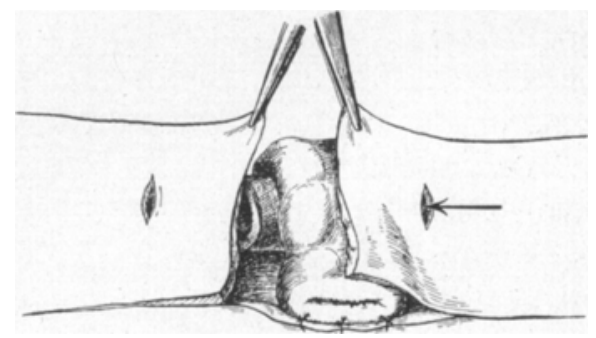

Fig. 5 .

schlauch und eine viereckige Wundfläche (Fig. 3). Auf diese wurde nun der Darm heruntergeklappt und mit einigen Nähten fixiert (Fig. 4). Nun handelte es sich darum, die Darmschlinge mit Haut zu bedecken. Zur Erreichung dieses $Z$ weckes wurden zwei Wege beschritten: a) Umschneidung und Mobilisierung der Haut über dem Darme, Vernähung und Einschneiden je eines seitlichen Knopfloches zum Einnähen der Hautschlauchenden (Fig. 5). b) Mobilisierung der Haut von oben her, Herunterziehen des gestielten Lappens über den Darm. Fixation durch Naht (Fig. 6). 
Natürlich ließe sich die Hautdeckung auch noch auf andere Weise erzielen. Die Menge der zu der Plastik zur Verfügung stehenden Haut wird die Technik im einzelnen bestimmen.

Bei dem ersten derartig operierten Fall ergab sich, wie vermutet, durch Zusammendrücken des Darmes zwischen einem im Hautschlauch befindlichen Metallstab - und einer von außen drückenden Pelotte völlige Kontinenz. Durch Variieren der Kompression konnte von dem Patienten ein Mittelwert des Druckes gefunden werden, der sowohl festen wie flüssigen Stuhlgang vollkommen zurückhielt, Flatus aber entweichen ließ. Das Resultat der Operation war ausgezeichnet. Dem Patienten, einem hohen Staatsbeamten, wurde wieder die Möglichkeit gegeben, seinen Dienst zu versehen und auch seinen repräsentativen Pflichten im vollen Umfange zu genügen. Nach monatelanger Beobachtung stellte sich aber heraus, daß es nicht ganz ohne Bedenken ist, den Darm zwischen einem im Hautschlauch befindlichen Metallstabe und einer Pelotte unelastisch zusammenzudrücken. Vielleicht hatte die fehlende Sensibilität an der Stelle der Plastik den Patienten, der auch den Flatus zurückzuhalten wünschte, verleitet, die Kompression des Darmes zu übertreiben. Nach der angeführten Zeit stelite sich im Haut-

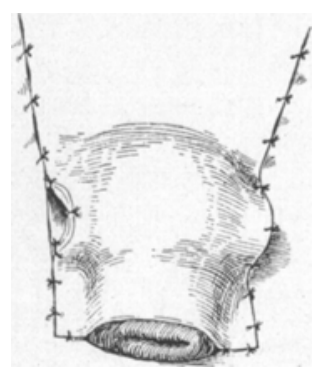

Fig. 6. schlauch eine Druckstelle heraus, die zu einer Kotfistel führte und das Operationsresultat in Frage stellte. Durch einen weiteren Eingriff gelang es, wieder Kontinenz zu erzielen. Näheres darüber am Schlusse der Arbeit.

Bei einer weiteren Patientin, deren Anus iliacus nach der Amputatio recti in oben beschriebener Hautschlauchplastik angelegt war, wurde die starre Kompression vermieden. Den Metallstab im Hautschlauch ersetzte ein Gummischlauch, dessen Höhlung zur Vermehrung der Zugfestigkeit ebenfalls mit Gummi ausgefüllt war. Die Außenpelotte wurde mit einer Bruchbandfeder in Verbindung gebracht und also von einem älteren Prinzip Gebrauch gemacht, das darin besteht, den Darm zwischen Außenhaut und Fascie bzw. Muskelplatte zu komprimieren. Durch zwei Durchbohrungen der Pelotte wurde ein Ende des Gummischlauches 
hindurchgesteckt und konnte hier mit einer Klemmschraube fixiert werden. Die Pelotte' $\mathrm{sa}$ ß somit unverschieblich fest. Durch mehr oder weniger starkes Anziehen des Gummischlauches konnte der Druck auf den Darm vermehrt oder vermindert werden. Die Stärke der Kompression des Darmes zwischen Pelotte und Muskelplatte bestimmte die Bruchbandfeder. Die Schraffierungen der Skizze 7 lassen die Druckzonen erkennen. Eng schraffiert ist die Kompressionszone zwischen Pelotte und Gummi im Hautschlauch, schwächer schraffiert die Druckzone zwischen Pelotte und Bauchmuskelplatte. Durch die geschwungene Form der Pelotte wird eine

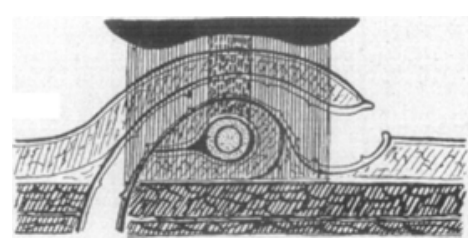

Fig. 7.

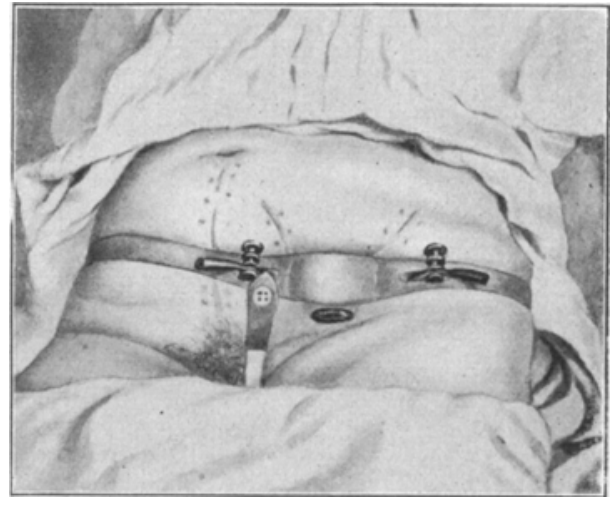

Fig. 8 .

möglichst gleichmäßige Kompression erstrebt. Das naturgetreu gezeichnete Bild 8 zeigt die Pelotte in situ.

Die Kontinenz der Patientin mit diesem Apparat war eine vollständige. Beschwerden traten nicht auf. $\mathrm{Zu}$ erwähnen ist noch, daß durch Variationen des Druckes eine Mittelstellung gefunden werden konnte, die eben noch Darmgase entweichen ließ. Die Hauptfrage war aber, wie die Plastik und damit die Kontinenz sich in Zukunft verhalten würde. Ich glaube, daß man jetzt, nachdem die Frau den Apparat über I3 Monate trägt, berechtigt ist, ein abschließendes Urteil zu fällen. Diese Dauerbeobachtung verdient besonders hervorgehoben zu werden, da in der Mehrzahl der Arbeiten, die dieses Thema besprechen, wohl über die Kontinenz unmittelbar nach der Operation, nicht aber über spätere Beobach- 
tungen berichtet wird. In dem mitgeteilten Falle besteht volle Kontinenz unverändert fort. Die Plastik selbst hat sich nicht verändert. Insbesondere haben sich keinerlei Ernährungsstörungen am Hautschlauch eingestellt. Es sei, wie die Frau sagt, ,alles in Ordnung". Sie ist mit der Pelotte imstande, beschwerdefrei ihren Landhaushalt $\mathrm{zu}$ besorgen und bleibt dabei vollkommen sauber.

Die Seltenheit dieser Dauererfolge rechtfertigt ihre Mitteilung. In der Literatur habe ich ähnliche Fälle einer über Monate bestehenden vollkommenen Kontinenz des künstlichen Afters nicht finden können.

Kurz erwähnt sei noch, daß die Möglichkeit besteht, die Haut-

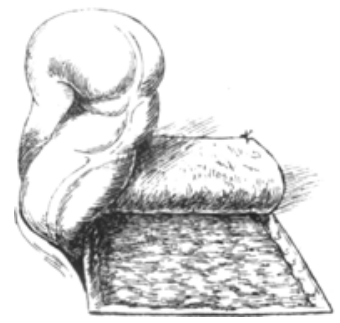

Fig. 9.

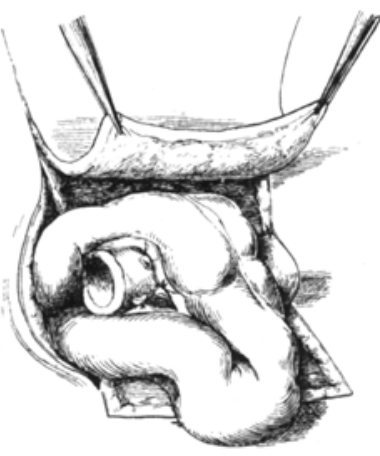

Fig. IO.

schlauchplastik auch bei der Anlegung des doppelläufigen Kunstafters anzuwenden. In diesem Falle müßte der Hautschlauch seitlich von der Herausleitungsstelle der Darmschlinge aus der Bauchhöhle angelegt worden, sonst in gleicher Weise wie bisher (Fig. 9). Die Darmschlinge würde dann derart auf den Hautschlauch zu liegen kommen, daß sie seitlich abgeknickt ist und daß das mediale Ende des Hautschlauches durch eine Öffnung des Mesosigma zutage tritt. Auf diese Weise wird das Ziel erreicht, die Darmschlinge über dem Hautschlatich zu lagern. Die Hautdeckung wäre dann wieder auf die beiden oben beschriebenen Weisen möglich. Fig. Io zeigt die Hautdeckung mit einem von oben her mobilisierten Hautlappen. Ich glaube, daß die beiden Skizzen das Verfahren hinreichend erläutern.

E. Unger und E. Schwabe haben, veranlaßt durch die 
Mitteilung meiner Leichenversuche, kürzlich eine Hautschlauchmethode zum Verschluß des künstlichen Afters versucht und in drei Fällen beim doppelläufigen Anus angewandt. Sie gingen folgendermaßen vor: Zwischen einer Stelle unterhalb des Nabels und dem linken Rippenbogen wurde eine Hautbrücke gebildet, und die Ränder zu einem Hautschlauch vernäht. Die Colonschlinge wurde am lateralen Schnittrande herausgeleitet und durch einen kleinen Schlitz ihres Mesenteriums die Mitte des Hautschlauches durchgeführt. Lateral von dem Hauptschnitt wurde ein Knopflochschnitt angelegt, zwischen Haut und Fascie ein Kanal zu dem Hauptschnitt gebohrt, der Hautschlauch durchgezogen, in seiner Mitte, quer zum Verlauf, zur Hälfte gespalten und mit den Knopflochwundrändern vernäht. Durch Verschiebung der Bauchhaut erfolgte die Hautdeckung der Darmschlinge, welche gleichsam auf zwei Hautschläuchen ritt. Beide Kanäle heilten gut ein und ließen sich mit Instrumenten bis zu Kleinfingerstärke ohne Schwierigkeit sondieren. Die Patientin starb an ihrem Grundleiden, ohne daß eine Pelotte erprobt werden konnte. In einem zweiten Falle wurde statt des Brückenlappens ein einfach gestielter Hautlappen zur Bildung des Hautschlauches benutzt; er wurde nekrotisch. In dem letzten Falle, bei dem der Hautschlauch in früheren Operationsnarben ruhte, führte die Spannung ebenfalls zur Nekrose.

Es sei hervorgehoben, daß bei unserem Vorgehen, welches wir oben ausführlich beschrieben haben, sich niemals eine $\mathrm{Ne}$ krose von Stellen der Hautplastik ergeben hat. Weil nur e in Hautschlauch angelegt wird, hat man für diesen mehr Material zur Verfügung und kann ihn demgemäß auch weiter anlegen. Der Meinung der Autoren, daß bei solchen Verfahren reichlich bewegliche, gut ernährte Haut zur Verfügung stehen muß, können wir beipflichten. Je weiter die Kanäle sind, um so leichter ist die Reinigung und Hautpflege durchzuführen und um so eher werden sich Zufälle, wie bei dem ersten von uns operierten Falle, vermeiden lassen. Bei diesem Patienten war, wie bereits erwähnt, durch zu energische und unelastische Kompression des Darmrohres eine Kotfistel entstanden. Da das Prinzip, durch das Kirs ch ner dem Patienten wieder zur Kontinenz verhalf, auch in einigen anderen Fällen primär zur Erzielung der Kontinenz mit Erfolg benutzt wurde, sei es hier mitgeteilt. 
Es wurde der die Bauchwand S-fürmig durchsetzende Darm nach Umschneidung der ihn bedeckenden Haut von der Unterlage wieder gelöst. Er passierte die Bauchwand nun senkrecht und war auf seiner kranial gerichteten Hälfte mit Haut bedeckt. Durch eine Brückenlappenplastik wurde die Hautdeckung der unteren Hälfte des Darmrohres erzielt. Das Ergebnis war ein penisartiges, etwa $5 \mathrm{~cm}$ langes Gebilde, das allseitig mit Haut bedeckt war, und im Innern den an der Spitze ausmündenden Darm beherbergtc (Fig. I I). Durch Komprimierung ergab sich auch hier Kontinenz. Das Verfahren ähnelt in seinem Endergebnis dem von Lauenstein und Bond mitgeteilten. Zur Beendigung dieser Plastik sind mindestens 2 Sitzungen erforderlich, im Gegensatz zu der oben geschilderten Hautplastik, die in einem Zuge, im Anschluß an

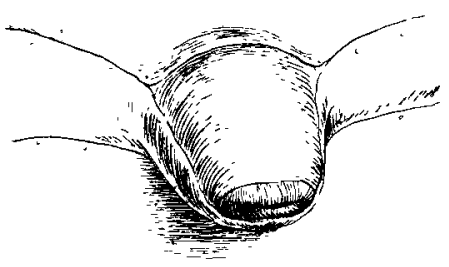

Fig. II. die Amputatio recti, zu vollenden ist. Unsere Erfolge waren auch auf diesem Wege erfreuliche. Besonders der erwähnte hohe Staatsbeamte war uns dafür dankbar, daß er zum zweiten Male die verlorene Kontinenz wiedererhielt. Hautschädigungen konnten auch bei starrer Kompression dadurch vermieden werden, daß die Klammer, die das penisartige Gebilde zusammendrückte und dadurch den Darm verschloß, an wechselnde Stellen angelegt wurde. Weiterhin wurde die besondere Sauberkeit der Stuhlentleerung gerühmt. Die Patienten konnten nach Abnahme der Klemme beim Einsetzen der Darmperistaltik den von Haut umhüllten Darmteil in die Hand nehmen und die Defäkation ohne die geringste Beschmutzung der Haut durch Vornüberbeugen über die Abortöffnung oder eine vorgehaltene Schale vornehmen.

Zieht man das Fazit unserer Kontinenzoperationen, so gelangt man $\mathrm{zu}$ einem durchaus günstigen Gesamturteil. Ablehnende Urteile über Kontinenzoperationen überhaupt, wie sie im Anfange erwähnt wurden, stehen mit den Erfolgen unserer neuen Verfahren in Widerspruch. Wenn auch das Ziel jeder Operation wegen Rektum-Karzinom sein wird, den Sphincter zu erhalten und wenn es auch nicht gelungen ist und wohl auch leider nicht gelingen wird, einen echten Sphincter zu bilden, so ist es 
I38 KuRtZAhn, Verfahren zur Erzielung der Kontinenz bei Anus praetern.

doch auf den angegebenen Wegen möglich, den Patienten durch eine technisch einfache, gefahrlose Operation die Kontinenz wieder zu schenken und ihnen damit die Arbeitsmöglichkeit und Lebensfreude zu erhalten.

\section{Literaturübersicht.}

I. B a $\mathfrak{u}$ er , A. u. S ch mi e d e $\mathbf{n}$, Kontinenzbestrebungen. Ergebn. d. Chir. 1912,4 .

2. B o nd, zit. nach Schmieden, Uber Sphincterplastik am Darm. Ergebn. d. Chir. u. Orth., Bd. 4.

3. Carl, Anus praeternaturalis definitivus. Bruns' Beitr. z. khn. Chir., Bd. 86, H. I. Daselbst weitere Literaturangaben.

$4^{\prime}$ Föderl, Beitrag zur Chirurgie des Rektums und des Uterus. Wiener klın. Wochenschr. 1894, Nr. I4, I5, I7.

5. Goebel1. Zeitschr. f. Chir. 1921, H. 13, S. 452.

6. Hacker, v., Kolostomie mit Sphincterbildung aus dem linken Musc. rect. abdomin. Beitr. z. klın. Chir, Bd. 23, S. 628 .

7. Hochenegg, zit. nach Schmieden.

8. J enkel, Zur Behandlung der Incontinentia alvi. Zeitschr. f. Chir. I921, H. 3 , S 45 I.

9. Kirschner. Chrurgenkongreß 1920 .

Io. K ü m mell. Zeitschr. f. Chir. I92I, H. I3, S. 452.

II. Kurtzahn, Eine neue Moglichkeit der Bildung eines künstlichen Sphincters. Deutsche med. Wochenschr. I920, Nr. I7.

I 2 - Zur Erzielung der Kontınenz bes Anus praeternaturalis. Deutsche med. Wochenschr. I920, Nr. 20.

13. L. a u enstein, Zur Frage der Anlegung und Funktion des künstlichen Afters. Zentralbl. f. Chir. 1894, Nr. 45.

I4 L e n n a nder, zit. nach Schmieden.

15. Lieblein, Zur Statistik und Technik der Radikaloperationen des Mastdarmkrebses Beitr. z. klin. Chir, Bd.33, S. 464.

16 Poppert, Zur Frage der Erhaltung des Schließmuskels nach salsraler Rektumexstirpation. Münchner med. Wochenschr. I906, Nr. 3 I.

17. Rotter, Wiederherstellung der Kontinenz. Arch. f. klin. Chir. I9I2, 98 , HI. I.

18. Ry dygier, Zur Bildung eines künstlichen Sphincter anı. Zentralbl. f. klin. Chir. I894, Nr. 45.

19. Scl mieden, Uber Sphincterplastik am Darm. Ergebn. f. Chir. u. Orth., Bd. 4. Daselbst weitere Literatur.

20. Schoemaker, Plastischer Ersatz des Sphincter ani. Verh. d. Deutschen Ges. f. Chir. I909, zit. nach Schmieden.

21. Unger, E. u. E. S $\mathrm{Chwabe,} \mathrm{Eine} \mathrm{Hautschlauchmethode} \mathrm{zum} \mathrm{Ver-}$ schluß des kunstlichen Afters. Deutsche med. Wochenschr. I92 I, Nr. 2 I.

22. Wit $z$ e l, Zur Indikation und Technik der Kolostomie und Enterostomie. Zentralbl. f. Chir. I 894 , S. 937. 